\title{
Limitaciones tecnológicas de la tomografía por emisión de positrones (PET) para pequeños animales de laboratorio
}

\author{
J.J. Vaquero, M. Desco \\ Laboratorio de Imagen. Medicina y Cirugía Experimental. Hospital General Universitario Gregorio Marañón. Madrid.
}

\begin{abstract}
Resumen.-La visualización y cuantificación de la función de determinados órganos en animales de laboratorio mediante PET está demostrando ser una herramienta de gran relevancia para la caracterización del fenotipo de animales transgénicos y noqueados, en el estudio de modelos de enfermedades humanas, así como para el descubrimiento y desarrollo de nuevos medicamentos y sondas bioquímicas. Para poder utilizar la PET en animales de laboratorio de un modo análogo al que se aplica en humanos es necesario contemplar el factor de escala en el tamaño del vóxel así como mantener una similar estadística de contaje. En este trabajo se apuntan los problemas que estos requerimientos representan tanto para el diseño técnico de los tomógrafos como para la realización de los experimentos, y desde esta perspectiva se analizan las soluciones tecnológicas más relevantes. Finalmente, se comentan brevemente algunas características de sistemas disponibles hoy comercialmente (microPET y FOCUS, HiDAC, eXplore VISTA, MOSAIC, YAP-(S)PET y $r P E T)$.
\end{abstract}

\section{TECHNICAL LIMITATIONS OF THE POSITRON EMISSION TOMOGRAPHY (PET) FOR SMALL LABORATORY ANIMALS}

Abstract.-Visualization and quantification of organ function by PET in small laboratory animals is becoming an outstanding tool for characterization of phenotype of transgenic and knock-out animals, for the study of animal models of human diseases, and for the development of new therapeutic drugs and diagnostic biochemical probes. To be able to make use of the PET with small laboratory animals in the same way as it is operated with humans it is necessary to account for the volumetric scale factor as well as for the requirement of maintaining the counting statistics. This work sketches the problems that these requirements represent for the technical design of the scanners and for the execution of the experiments. Finally, some characteristics of commercially available scanners (microPET/ FOCUS, HiDAC, eXplore VISTA, MOSAIC, YAP-(S)PET and rPET) are briefly discussed.

\section{PRESENTACIÓN}

Los últimos avances en instrumentación para imagen médica han conducido al diseño y desarrollo de sistemas de imagen molecular especialmente dedicados a la obtención de imágenes de pequeños animales de laboratorio, imposibles de conseguir con la instru- mentación diseñada para humanos ${ }^{1,2}$. Estos equipos se usan fundamentalmente para investigación traslacional en biología molecular y medicina; se da la circunstancia de que el mayor número de modelos de enfermedades humanas, tanto en neurología como en cardiología y oncología, se ha desarrollado sobre roedores, debido al buen conocimiento de estos modelos y su bajo coste ${ }^{3}$. La evolución de la genómica y de la biología molecular, así como la investigación con animales modificados genéticamente ${ }^{4}$ ha impulsado el desarrollo de sistemas no invasivos de imagen para pequeños animales con el fin de poder realizar estudios longitudinales en periodos de tiempo relativamente largos, experimentos en los que es necesaria la supervivencia del animal ${ }^{5,6}$. Sin embargo la disponibilidad y el acceso a este tipo de sistemas se ven limitadas por la dificultad tecnológica y el coste que entraña su diseño y construcción.

Las modalidades que actualmente despiertan más interés para imagen animal son dos: PET (positron emission tomography $)^{7}$ y CT (computerized tomography). La PET es probablemente la técnica más potente para el estudio in vivo de procesos biológicos en animales de laboratorio, al permitir mediciones funcionales y cuantitativas de forma no invasiva, así como la realización de estudios longitudinales. Esta modalidad de imagen permite estudiar, visualizar y cuantificar múltiples procesos bioquímicos y fisiológicos tales como metabolismo glicídico, tasa de síntesis proteica, proliferación celular, actividad enzimática, tasa de consumo de oxígeno, metabolismo $\beta$-oxidativo, $\mathrm{pH}$ intracelular, flujo sanguíneo, transmisión de señales, expresión génica y su regulación, entre otros ${ }^{8}$.

Idealmente desearíamos disponer de un equipo PET muy sensible capaz de cuantificar pequeños cambios, junto con una resolución elevada para facilitar una localización lo más precisa posible. Sin embargo, el estado actual de la técnica ${ }^{9-14}$ obliga a los diseñadores de tomógrafos a optar por soluciones de compromiso entre sensibilidad y resolución, dando 

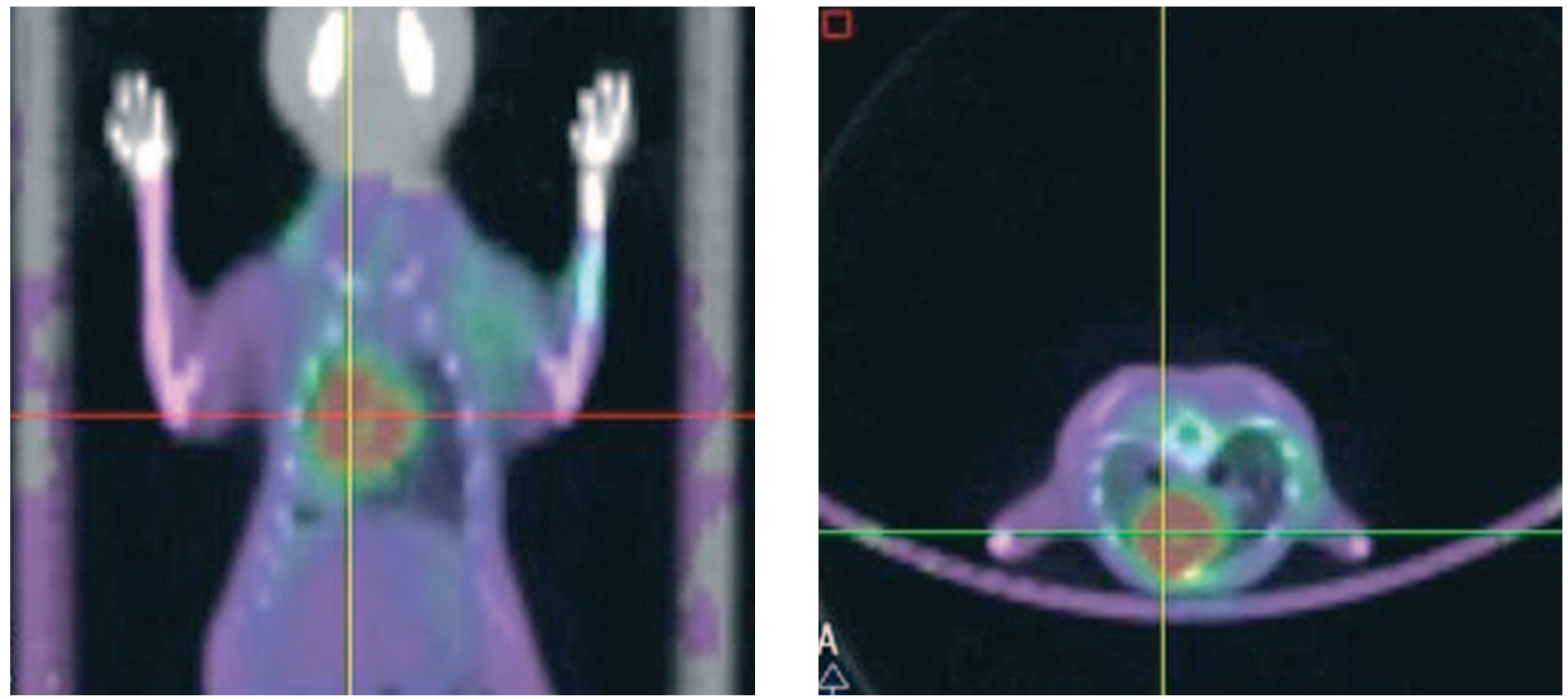

como resultado sistemas de características muy distintas, cuya comparación resulta especialmente dificultosa. Así como para los equipos de uso en humanos existe una normativa que permite a un usuario realizar una comparación en términos equiparables (el estándar NEMA UN 2-1994 y el UN 2-2001) ${ }^{15-17}$, para los sistemas de uso en animales todavía no se ha desarrollado un protocolo equivalente.

Una de las dificultades que debe afrontar la imagen PET para trabajar a una escala adecuada a pequeños animales de laboratorio es su relativamente baja resolución espacial, que dificulta la identificación de las estructuras y obliga en muchos casos a la localización de "marcas" que permitan reconocer con precisión las activaciones visualizadas. La denominada "captación no específica" puede ayudar a encontrar referencias anatómicas pero, según los trazadores van siendo más selectivos, resulta cada vez más difícil identificar estructuras que sirvan de referencia. De ahí surge la necesidad de utilizar también otras modalidades de imagen estructural que aporten esa información anatómica que falta, como, por ejemplo el CT, que incluso resulta útil para realizar las correcciones necesarias en el procesamiento de los datos PET $^{18,19}$ (fig. 1).

Otro problema que surge en la PET de alta resolución en animales de laboratorio es que se hace imperativo aumentar la relación dosis/masa, con el objeto de mantener una cifra de cuentas acumuladas por vóxel similar a la que se alcanza en estudios con huma-

nos. Dada la dificultad de incrementar la actividad específica del radiofármaco, es necesario aumentar el volumen inyectado. Dicho aumento supone la aparición de captación no específica en otros tejidos, lo que en la imagen se traduce por la existencia de un "fondo" que deteriora la relación señal/ruido. Este problema no sólo dificulta la detección de alteraciones sutiles, sino que puede afectar a la fiabilidad de la cuantificación si no se aplican las oportunas correcciones $^{1,20}$. Además, se añade la posible saturación de los sistemas que se pretende visualizar, especialmente cuando se está estudiando densidad de receptores; en otros sistemas, principalmente los basados en enzimas, es más difícil llegar a producir esta saturación. Los parámetros técnicos del sistema PET juegan, por tanto, un papel mucho más crítico en imagen animal que en imagen clínica.

A continuación se repasan algunos de los muchos factores técnicos y experimentales que determinan las prestaciones alcanzables por estos sistemas en la práctica.

\section{LIMITACIONES DE LA TÉCNICA PET EN PEQUEÑOS ANIMALES DE LABORATORIO}

Para entender la dificultad que supone el traslado de la tecnología PET a la escala del animal de laboratorio (rata o ratón), conviene analizar con detalle las 
consecuencias de dicho escalado sobre la visualización y cuantificación de la distribución de un determinado trazador; idealmente se requeriría un sistema de imagen con una resolución espacial entre cinco y diez veces mejor que la que actualmente ofrecen los sistemas clínicos ${ }^{1}$. Esta cifra está todavía lejos de lograrse y por lo tanto hay que analizar los factores que limitan la resolución alcanzable y qué otras consecuencias sobre el planteamiento de los experimentos podría acarrear el acercarse a los límites teóricos de resolución.

Los factores limitantes mencionados se pueden clasificar en tres categorías generales, que estudiaremos a continuación:

1. Limitaciones físicas e instrumentales.

2. Limitaciones experimentales.

3. Limitaciones farmacológicas.

Obtener imágenes de alta resolución espacial y cuantitativamente precisas requiere contemplar las tres categorías, dado que todas contribuyen de forma significativa al deterioro de la calidad de imagen.

\section{Limitaciones físicas e instrumentales}

Bajo este epígrafe agrupamos las limitaciones derivadas de la complejidad del fenómeno físico subyacente a la imagen $\mathrm{PET}^{9}$, así como las dependientes de la instrumentación asociada.

\section{Limitaciones físicas}

El denominado rango del positrón introduce una primera incertidumbre sobre el lugar preciso en que se encuentra el trazador, que no coincide con el punto en que se ha producido la aniquilación positrón-electrón y consiguiente generación del par de fotones gamma. Dicho rango se caracteriza por la distancia media que recorre el positrón antes de aniquilarse, y depende del isótopo y de la materia en la que se encuentre ${ }^{21,22}$.

Otro efecto a considerar es la no colinealidad de la emisión de los fotones gamma producto de la aniquilación; los 180 grados teóricos de diferencia con que se emiten dichos fotones no son exactamente tales en función del principio de conservación de la cantidad de movimiento: el positrón mantiene parte de su momento y de su energía cinética justo antes de la aniquilación, lo que origina una no colinealidad de alre- dedor de $0,25^{\circ}$, que, dependiendo del diámetro del escáner, pude llegar a traducirse en un emborronamiento de hasta $2 \mathrm{~mm}$ para sistemas de humanos, o $0,3 \mathrm{~mm}$ en sistemas de pequeño diámetro para animales $^{21,22}$.

\section{Cristales de centelleo y el problema de la profundidad de la interacción}

Los detectores PET más comunes consisten en un cristal de centelleo y un fotodetector (tubos fotomultiplicadores o semiconductor) cuyo tamaño mínimo viene impuesto por la tecnología de fabricación de los mismos. Este tamaño determina la resolución intrínseca del conjunto detector, $y$, aun cuando fuese posible reducirlo, esto reduciría el volumen de material centelleador y, por tanto, la probabilidad de que el rayo gamma de alta energía $(511 \mathrm{keV})$ sea absorbido y convertido en fotones de luz ${ }^{23,24}$, disminuyendo la sensibilidad. Por eso se buscan materiales con una alta densidad efectiva para los rayos gamma. El BGO ha sido tradicionalmente el centelleador por excelencia para las aplicaciones PET, pero en los últimos años han aparecido nuevos cristales que producen más luz y cuya duración del pulso centelleador es más breve, lo que permite acelerar el proceso de detección, reducir los problemas de pile-up en altas tasas de conteo, e incluso reducir la ventana de coincidencia, lo que redunda en una reducción de eventos aleatorios. La tabla 1 muestra una lista de materiales recientes utilizados para la detección de rayos gamma y con potenciales aplicaciones en PET.

Una alternativa de diseño de un tomógrafo de alta resolución y alta sensibilidad es la de acercar los detectores a la muestra tanto como sea posible. En sistemas con geometría anular, este acercamiento del anillo de detectores al campo de visión acentúa de forma notable el error de la profundidad de la interacción (DOI, depth of interaction). Este error de paralaje consiste en que el cristal presenta una sección efectiva mayor para fuentes colocadas cerca del borde del FOV, y, por lo tanto, las líneas de respuesta (LOR) correspondientes son de mayor grosor (fig. 2). En sistemas que utilizan detectores planos este error es bastante menos importante; para sistemas anulares una solución puede ser disminuir el tamaño en la dirección radial (altura del cristal), pero esto incidiría negativamente en la sensibilidad del sistema. Se han propuesto otras alternativas, como los sistemas basados en cristales apilados (phoswich) ${ }^{25,26}$ o en anillos concén- 
Tabla 1

MATERIALES UTILIZABLES PARA CRISTALES DE CENTELLEO

\begin{tabular}{|c|c|c|c|c|c|c|c|c|c|c|}
\hline & $\mathrm{NaI}$ & $B G O$ & GSO & LSO & LYSO & $L P S$ & LUYAP & $\operatorname{LaBr} 3$ & LuI3 & $M L S$ \\
\hline Luminosidad (ph/MeV) & 38 & 8,2 & 10 & 28 & 37 & 26 & 12,5 & 60 & 47 & 26,6 \\
\hline Energy Resol, & 7 & 12 & 9 & 10 & 8 & 10 & 8 & 3 & $\leq 5 \% ?$ & \\
\hline Desidad efectiva Z & 51 & 75 & 59 & 66 & 64,5 & 63,8 & 64,9 & & & 64 \\
\hline Const. de tiempo (ns) & 230 & 300 & $30 \sim 60$ & $35 \sim 45$ & $45 \sim 60$ & 38 & 25,200 & 25 & 30 & 38 \\
\hline Densidad (g/cc) & 3,67 & 7,13 & 6,7 & 7,4 & 7,3 & 6,2 & 7,4 & 5,3 & 5,6 & 7,35 \\
\hline Longitud de atenuación (mm) & 25,6 & 11,2 & 13,8 & 11,4 & 12 & 15 & 13 & 22 & 18 & 11,6 \\
\hline Fotofracción & 0,17 & 0,43 & 0,25 & 0,34 & & 0,31 & 0,27 & 0,14 & 0,29 & \\
\hline Longitud de onda (nm) & 410 & 480 & 440 & 420 & 400 & 385 & 390 & 370 & 470 & 420 \\
\hline Índice refracción & 1,85 & 2,15 & 1,85 & 1,82 & 1,82 & & 1,94 & & & 1,82 \\
\hline ¿Radioactivo? & No & No & No & $\mathrm{Si}$ & $\mathrm{Si}$ & $\mathrm{Si}$ & $\mathrm{Si}$ & No & $\mathrm{Si}$ & $\mathrm{Si}$ \\
\hline ¿Higroscópico? & $\mathrm{Si}$ & No & No & No & No & No & No & $\mathrm{Si}$ & $\mathrm{Si}$ & No \\
\hline
\end{tabular}

tricos ${ }^{27}$. El problema se manifiesta como una pérdida de la resolución al alejarse del centro del FOV, y la rapidez con que se deteriora dicha resolución depende del diseño del sistema, y justifica la gran importancia de conocer la resolución a diferentes distancias del centro para comparar las prestaciones de diferentes equipos.

\section{Tecnología de los detectores}

Las principales características los detectores para imagen PET son que permita definir con la mayor precisión geométrica las posibles LORs, y que a su vez sea sensible y detecte un gran porcentaje de los fotones que alcancen dicho detector ${ }^{28,29}$. Para lo primero se precisan cristales pequeños y detectores sensibles a posición, con un fino muestreo espacial ${ }^{30}$. Para aumentar la sensibilidad esos mismos cristales deberían ser grandes y densamente empaquetados en una configuración geométrica compacta.

Si se define la resolución como la capacidad del sistema para percibir separados dos objetos pequeños próximos ${ }^{20,31}$, en la imagen PET se hace necesario distinguir tres tipos de resolución espacial: radial, tangencial y axial. Las resoluciones radial y tangencial se miden en el plano de imagen, sobre perfiles radiales y tangenciales generados sobre la imagen que representa una fuente puntal, y midiendo su anchura a una altura mitad del máximo (FWHM, full width half maximum). La misma fuente puntual situada a diferentes distancias del centro da lugar a imágenes con distintos grados de emborronamiento (sistema espacialmente variante). Esto hace que sea

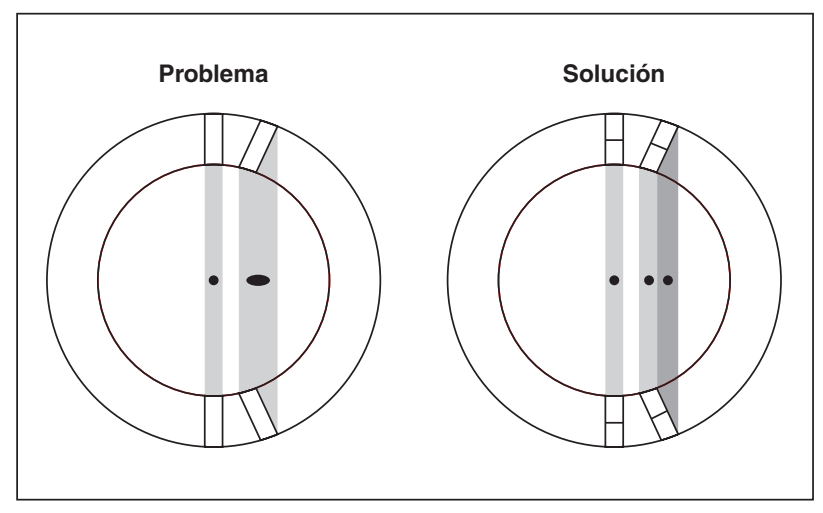

FIG. 2.-Efecto de la profundidad de la interacción en un sistema PET de anillo. A la izquierda se muestra la variación de sección de cristal vista por un punto cerca del borde del FOV frente a esa misma sección vista desde el centro. Una solución (derecha) es el uso de capas de cristales de centelleo distintos ("phoswich") que, al poderse diferenciar, reducen la sección efectiva de esas lineas de respuesta.

necesario medir las resoluciones radial y tangencial a diferentes distancias del centro de la imagen. La tercera medida de resolución es la axial; lo habitual en tomógrafos PET de animales es tener vóxeles isotrópicos, de forma que en el volumen final reconstruido la resolución es prácticamente la misma en los tres ejes del espacio.

En un intento de formalizar el efecto de los factores que influyen en la resolución en la imagen reconstruida $-R-$ se propuso la siguiente fórmula empírica $^{20}$ :

$$
R=a \cdot \sqrt{\left(\frac{d}{2}\right)^{2}+b^{2}+r^{2}+(0,002 \cdot D)^{2}}
$$


Tabla 2

ENERGÍAS Y RANGOS DEL POSITRÓN DE LOS ISÓTOPOS HABITUALMENTE UTILIZADOS EN PET

\begin{tabular}{lccccc}
\hline Isótopo & Vida media $\left(T_{1 / 2}\right)$ & Branching ratio $(\%)$ & Energía máxima de $\beta^{+}(\mathrm{MeV})$ & FWHM $(\mathrm{mm})$ & FWHM efectiva $(\mathrm{mm})$ \\
\hline${ }^{11} \mathrm{C}$ & $20,4 \mathrm{~min}$ & 99 & 0,96 & 0,13 & 0,92 \\
${ }^{13} \mathrm{~N}$ & $9,96 \mathrm{~min}$ & 100 & 1,2 & 0,17 & 1,35 \\
${ }^{15} \mathrm{O}$ & $2,05 \mathrm{~min}$ & 100 & 1,7 & 0,28 & 2,4 \\
${ }^{18} \mathrm{~F}$ & $1,83 \mathrm{~h}$ & 97 & 0,64 & 0,13 & 0,54 \\
${ }^{64} \mathrm{Cu}$ & $12,7 \mathrm{~h}$ & 19 & 0,65 & 0,13 & 0,54 \\
${ }^{68} \mathrm{Ga}$ & $1,14 \mathrm{~h}$ & 88 & 1,9 & 0,31 & 2,8 \\
${ }^{82} \mathrm{Rb}$ & $1,3 \mathrm{~min}$ & 95 & 3,4 & 0,42 & 6,1 \\
\hline
\end{tabular}

donde $d$ es el tamaño del detector, $b$ la precisión de la decodificación de la posición (dependiente del diseño del detector), $r$ el rango del positrón (tabla 2), $D$ la distancia entre detectores (que incluye indirectamente la influencia de la no colinealidad), y $a$ un factor de escala que depende del algoritmo de reconstrucción de la imagen, que varía entre 1.1 y 1.3 , tomado el valor 1.25 para la retroproyección filtrada con filtro de rampa.

El efecto del rango del positrón sobre la imagen, calculado en función de su valor medio, es pequeño. En la práctica, sí se observan diferencias de calidad de imagen cuando se obtienen imágenes con isótopos con energías del positrón muy distintas (tabla 2), lo que se explica por las largas colas de la distribución de valores del rango del positrón. Una descripción detallada de este efecto puede encontrarse en el artículo de Levin y Hoffman ${ }^{21}$.

Las componentes $d$ y $b$ dependen de la ingeniería del detector; la mayor parte de los sistemas actuales,

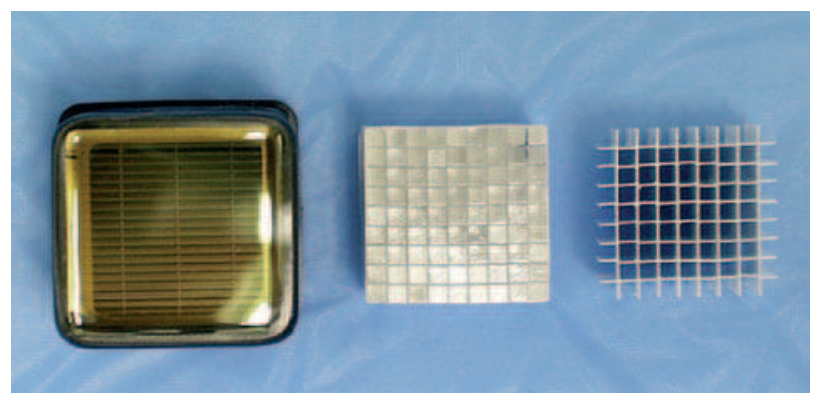

FIG. 3.-Elementos que componen un detector PET de alta resolución: a la izquierda el fotomultiplicador sensible a posición en el que se aprecia el fotocátodo (ventana de entrada con reflejos dorados) y la estructura de dínodos que preservan la información espacial. En el centro una matriz de cristal de LSO en la que se aprecian los cristales individuales, y a la derecha el soporte de reflectores que, además de servir como aislantes ópticos entre cristales, dan rigidez. mecánica al conjunto. tanto de humanos como de animales, se pueden clasificar en tres diferentes categorías, en función de la forma y el acoplamiento entre el cristal centelleador y el fotodetector: detectores "de bloque", de cristal segmentado y de canales independientes.

Los detectores denominados "de bloque" son los más comúnmente utilizados en sistemas clínicos de humanos por su menor coste y sencilla fabricación en grandes cantidades. Utilizan matrices de cristales de gran tamaño (más de $4 \mathrm{~mm}$ de lado) acopladas a varios dispositivos fotodetectores conectados a una electrónica denominada lógica de Anger, que permite calcular el punto más probable de la interacción gamma-cristal ${ }^{32,33}$. El principal problema de estos detectores es que, dado el gran tamaño del cristal, la luz se dispersa iluminando áreas grandes del detector, lo que introduce incertidumbre en la medida. Este problema está bien caracterizado en los sistemas de humanos, con resolución en el orden de los $4 \mathrm{~mm}$, pero empieza a ser difícilmente controlable en sistemas de alta resolución.

Para limitar este efecto se diseñaron detectores con cristales segmentados, se separados físicamente mediante láminas reflectoras que confinan la luz, y acoplados a fotodetectores sensibles a posición (fig. 3), lo que mejora la resolución intrínseca (resolución propia del detector; en la global del sistema intervienen más factores) respecto a la de los detectores de bloque $^{28,30,34-39}$. Estos diseños, sin embargo, tienen un coste más elevado, razón por la cual su uso se ha limitado a los sistemas de animales, con un número de detectores mucho menor que en los sistemas para humanos.

Los detectores del tercer tipo asocian un único cristal de centelleo con un detector y con un único canal electrónico, con objeto de evitar aún más este posible cruce de información entre vecinos ${ }^{27,40}$. En este caso, la resolución intrínseca alcanzable responde di- 
rectamente a la geometría del cristal, puesto que no hay dispersión de la luz de centelleo que pueda distorsionar la identificación de la detección. Los sistemas basados en esta tecnología tienen otra ventaja adicional en el hecho de que, al tratarse de canales paralelos independientes para cada detector, es posible procesar varios eventos simultáneamente. La contrapartida es el alto coste y complejidad, así como los problemas de "empaquetamiento" de los detectores, las necesidades de potencia eléctrica y la disipación del calor generado.

Por último, conviene considerar la capacidad del sistema para procesar eventos: una mayor velocidad contribuye a disminuir los problemas de coincidencias aleatorias (randoms, fotones que aparecen como coincidencias pero que en realidad provienen de distintas desintegraciones) y de saturación (pile-up, coincidencia de varios fotones que producen un solo pulso con información de energía errónea, contribuyendo a la pérdida de eventos válidos). Para ello se necesita que los cristales sean rápidos, al igual que la electrónica de adquisición de datos, que ha de ser de bajo tiempo muerto (los circuitos de coincidencia no pueden procesar más de un evento durante la ventana de coincidencia temporal, intervalo durante el cual parecen "muertos" para nuevos eventos).

\section{Reconstrucción de la imagen}

La última etapa en la formación de imagen es la reconstrucción tomográfica, y la contribución de este componente a la calidad del conjunto es también muy importante. Al comparar imágenes de distintos sistemas es preciso considerar en el tipo de algoritmo utilizado, ya que los métodos más potentes producen resultados llamativamente mejores, pero para hacer comparativas los valores de resolución deben medirse sobre imágenes obtenidas mediante reconstrucciones analíticas (fig. 4).

De un modo simplificado, podemos agrupar las técnicas más habituales de reconstrucción de imagen en dos grandes tipos: analíticas y estadísticas iterativas.

\section{Reconstrucción analítica}

Estas técnicas se consideran aproximaciones a una transformada de Radon inversa, procedimiento que permite recuperar una imagen a partir de sus proyecciones. En las últimas dos décadas se han desarrollado varios algoritmos de reconstrucción para imágenes
PET, similares a los utilizados para la reconstrucción en otras modalidades tomográficas. En general, se basan en el conocido algoritmo de retroproyección filtrada en diversas variantes según el filtrado se efectúe antes o después de la retroproyección y en el dominio de la imagen o de la frecuencia. Una consideración importante es si los métodos utilizados son 2D o 3D. Los algoritmos analíticos son bien conocidos y están caracterizados en el caso bidimensional, pero casi todos los sistemas de PET para animales adquieren información en 3D. Desperdiciar esta información 3D supondría una pérdida de sensibilidad inaceptable. La opción más sencilla para reconstruir es utilizar un método 2D después de realizar lo que se conoce como "rebinning" o reformateo, que consiste en reagrupar las líneas de respuesta oblicuas asignándolas a cortes directos paralelos, de tal modo que el problema se puede aún resolver en $2 \mathrm{D}$, rescatando sensibilidad. El inconveniente es que este rebinning introduce emborronamiento en la imagen reconstruida, principalmente en el eje axial, lo que nos enfrenta de nuevo al dilema sensibilidad vs. resolución. Hay varios métodos de rebinning: el más sencillo es el denominado Single Slice Rebinning (SSRB), que proporciona un compromiso entre resolución, sensibilidad y tiempo de reconstrucción. Uno de los más recientes y eficientes es FORE (Fourier Rebinning), que obtiene resultados casi tan buenos como los métodos realmente tridimensionales ${ }^{41}$.

Entre los métodos tridimensionales destaca la retroproyección filtrada $3 \mathrm{D}$, bastante más compleja que su contrapartida bidimensional y para la cual hay varias propuestas de implementación; la denominada 3DRP ${ }^{42}$

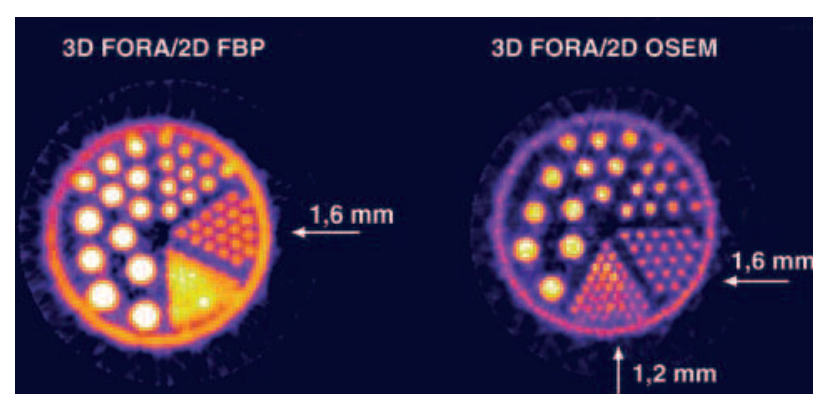

FIG. 4.-Comparación entre un método de reconstrucción analítico (FBP, izquierda) y un iterativo (2D-OSEM, derecha, 16 subsets, 15 iteraciones) para un fantoma tipo Derenzo de alta resolución (Micro DeLuxe, Data Spectrum Corporation, NC, USA). En ambos casos los datos $3 D$ se reformatean en $2 D$ usando el algoritmo FORE, y luego se procede a su reconstrucción por rodajas. Imágenes adquiridas en un sistema eXplore VISTA, GE Healthcare. 
es la más difundida, aunque hay otras más eficientes, sobre todo para geometría de detectores paralelos ${ }^{43}$. Estos métodos 3D son capaces de rescatar toda la sensibilidad del equipo al no desperdiciar ningún evento, sin por ello degradar la resolución, pero son significativamente más lentos que los $2 \mathrm{D}$, por lo que a veces se prefiere ir directamente a los algoritmos iterativos explicados a continuación, que, aunque son aún más lentos, permiten obtener mejor resolución.

\section{Reconstrucción iterativa}

La idea básica tras los métodos estadísticos de reconstrucción es formular el proceso de obtención de los datos PET como intrínsecamente aleatorio, en vez de ignorar este carácter, como hacen los métodos analíticos al aceptar como "buenos" los datos adquiridos, esto es, asumiendo que representan fielmente proyecciones del objeto. El modelado de los fenómenos físicos que limitan la resolución puede permitir recuperar parte de la resolución perdida por causa de esos factores.

Es conocido que la emisión gamma responde un proceso aleatorio de Poisson, pero, mientras que en el caso de tomografía por rayos $\mathrm{X}$ se puede alcanzar fácilmente un número de $10^{15}-10^{16}$ cuentas, es raro pasar de $10^{6}-10^{7}$ para un solo anillo de detectores PET, y menos aún en caso de estudios dinámicos. Por ello, la incertidumbre estadística (ruido) es mucho más importante en el caso del PET, y parece razonable que un método de reconstrucción capaz de incorporar la naturaleza estocástica del proceso de emisión produzca imágenes mejores que las técnicas convencionales.

Desde esta perspectiva, el problema de la reconstrucción de imagen en PET se puede considerar como un problema estadístico estándar de estimación con datos incompletos. Los datos adquiridos en PET se consideran incompletos por el hecho de que, aunque se conoce el par de detectores donde el evento fue registrado, no se conoce el origen del evento de aniquilación dentro de la línea de respuesta. En estadística hay un método iterativo general, conocido como maximización de la esperanza (expectation maximization, EM). El nombre de la técnica proviene del hecho que en cada iteración existe un paso de cálculo de expectativa (expectation step) que usa las estimaciones actuales de los parámetros para realizar una reconstrucción del proceso Poisson inobservable, seguida por un paso de máxima verosimilitud (maximum likelihood) que utiliza esta reconstrucción para revisar dichas estimaciones.
Un método iterativo de reconstrucción estadística tiene, por tanto, dos componentes esenciales: un modelo estadístico del sistema, que puede incorporar más o menos detalles del mismo, y un algoritmo tipo EM que conduce el proceso de iteración por el cual se recalcula el objeto "más probable" que daría lugar al conjunto de datos (proyecciones) realmente observado. El modelo calcula las proyecciones que produciría una distribución de trazador dada, incorporando normalmente la geometría del sistema, la sensibilidad de cada pareja de cristales, el ancho de cada línea de respuesta, etc. Puede incluso incorporar información sobre la física subyacente (rango del positrón, no colinealidad, etc.).

Los métodos iterativos pueden también ser 2D y $3 \mathrm{D}$, como los analíticos. Igualmente, para el caso 2D es necesario pasar por un rebinning inicial, lo que limita la calidad máxima que se puede conseguir respecto a la alcanzable por un método 3D.

La contrapartida de estos métodos iterativos es su alto coste computacional, que obliga a largos tiempos de cálculo o, alternativamente, al uso de redes de ordenadores trabajando en paralelo (clusters o, más modernamente, grids). Aunque también hay varios disponibles para equipos de uso humano, sobre todo en versión 2D, este tipo de reconstrucción resulta de mucho mayor interés para imagen animal, donde es crítica la necesidad de mejorar todo lo posible la resolución espacial.

Para ayudar a valorar los datos de los fabricantes respecto a la disponibilidad de métodos avanzados de reconstrucción es necesario destacar algunos aspectos. En lo referente a métodos analíticos, prácticamente todos los equipos ofrecen el conjunto completo de posibilidades $2 \mathrm{D}$ y $3 \mathrm{D}$, sin grandes diferencias, salvo en lo que puede referirse a la fidelidad cuantitativa de los algoritmos, difícil de valorar y sobre la que excepcionalmente se ofrece información. Respecto a métodos iterativos, su uso está cada vez más extendido y la oferta abarca múltiples modos de implementación. Sin embargo, el elemento crítico que más distingue unos de otros no es tanto el tipo de algoritmo iterativo utilizado (EM, OSEM, MAP, etc.) sino la calidad del modelo del sistema, qué parámetros incorpora y con qué eficiencia se almacena y maneja (datos éstos no ofrecidos por los fabricantes), lo que determina la calidad de imagen alcanzada y el tiempo de reconstrucción. No es raro encontrarse en la literatura imágenes de reconstrucciones iterativas cuya calidad es visiblemente peor que la de métodos analíticos clásicos, junto con 


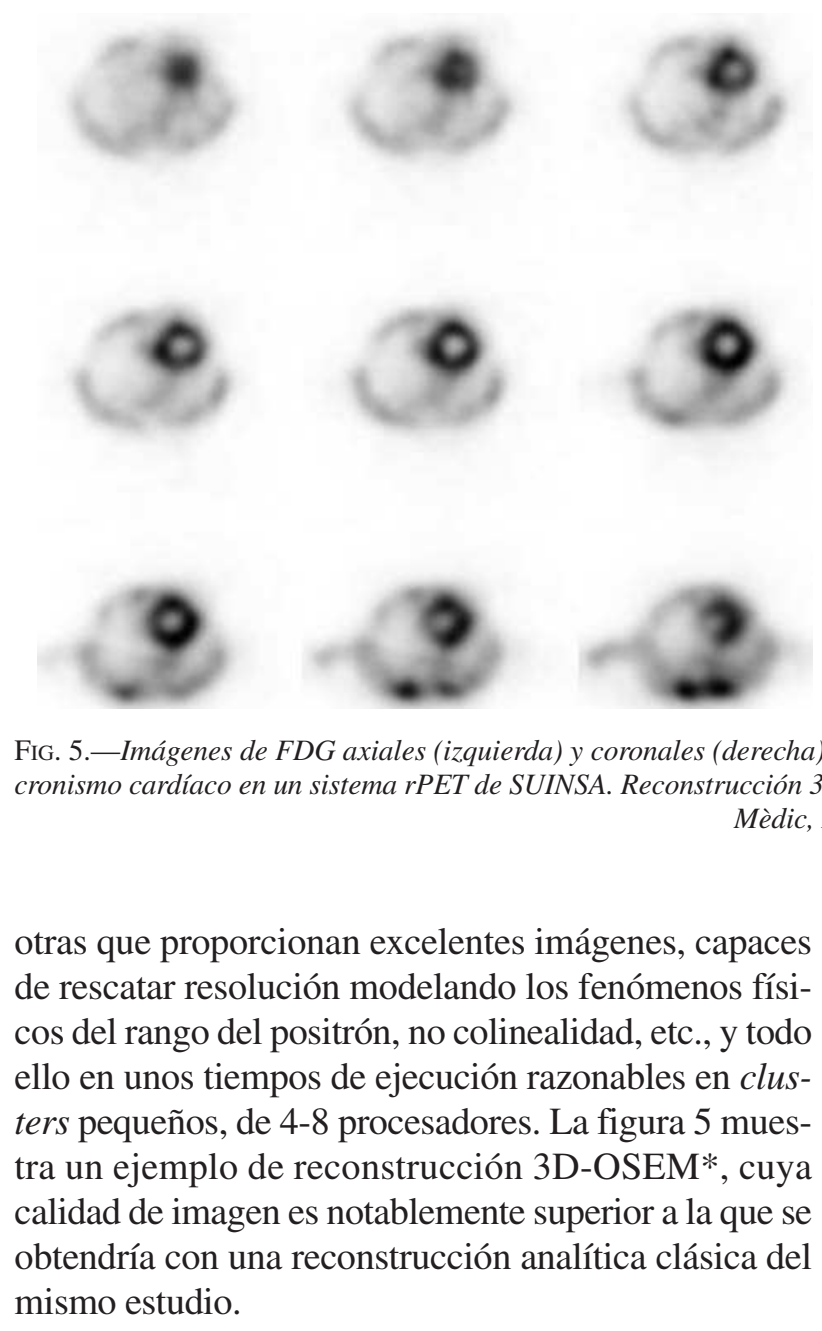

\section{Limitaciones experimentales}

La imagen PET animal también sufre deterioro por los mismos efectos que existen en los estudios clínicos con humanos, como son la dispersión (scatter), la atenuación de los rayos gamma o los movimientos involuntarios o inevitables (respiración, pulso cardíaco, etc.). Los problemas de atenuación son mucho menores, dado el menor tamaño de las muestras, si bien para una cuantificación muy precisa se aconseja realizar esta corrección. Sin embargo, la imagen de alta resolución es, lógicamente, mucho más sensible a los movimientos de la muestra.

Estos tres efectos están presentes en todas las realizaciones experimentales, $\mathrm{y}$, aunque pueden aliviarse aplicando algunos procedimientos tecnológicos (co-

*Obtenida sobre un cluster del Departamento de Física Atómica, Molecular y Nuclear, Universidad Complutense, Prof. J. M. Udías.

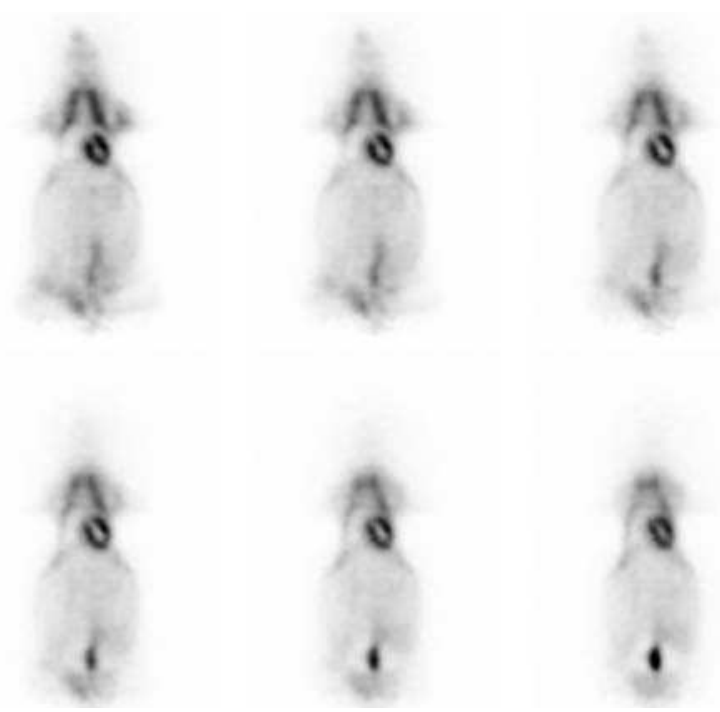

) del corazón de un ratón de 28 gramos. Los datos se adquirieron sin sinèdic, Barcelona).

rrección de la dispersión y de la atenuación, sincronismo fisiológico [gating]), es parte del arte del experimentador el poner los medios para disminuir el impacto de estos factores en la calidad de la imagen final utilizando procedimientos como una correcta calibración del sistema, la disminución de la actividad fuera del campo de visión, o la adecuada inmovilización de la muestra.

Dentro de las limitaciones experimentales, una tarea difícil de realizar, pero fundamental a la hora de hacer estudios dinámicos cuantitativamente precisos, es la estimación de la función de entrada, que idealmente debería ser medida en cada experimento. En un estudio PET cuantitativo, tan crítica como la imagen puede ser la medida de metabolitos marcados en sangre durante el desarrollo del experimento, lo que en humanos o en mamíferos de tamaño grande o mediano se hace mediante extracción de muestras de sangre. Sin embargo, una rata tiene un volumen de sangre aproximado de $20 \mathrm{ml}$, de los cuales es posible extraer entre un 10 y un $12 \%$ de forma segura durante un periodo de 60 a 90 minutos, que suele ser la duración de un experimento completo. Esta limitación del volumen de sangre a extraer dificulta las medidas, si bien algunos investigadores han diseñado ingeniosos métodos para poder llevarlas a cabo ${ }^{44}$. Las dificultades son aún mayores si el experimento se hace con ratones, dado que en ese caso apenas se puede disponer de 0,2 o $0,3 \mathrm{ml}$ de sangre, de los aproximadamente 2,6 ml que tiene el animal. En todos los ca- 


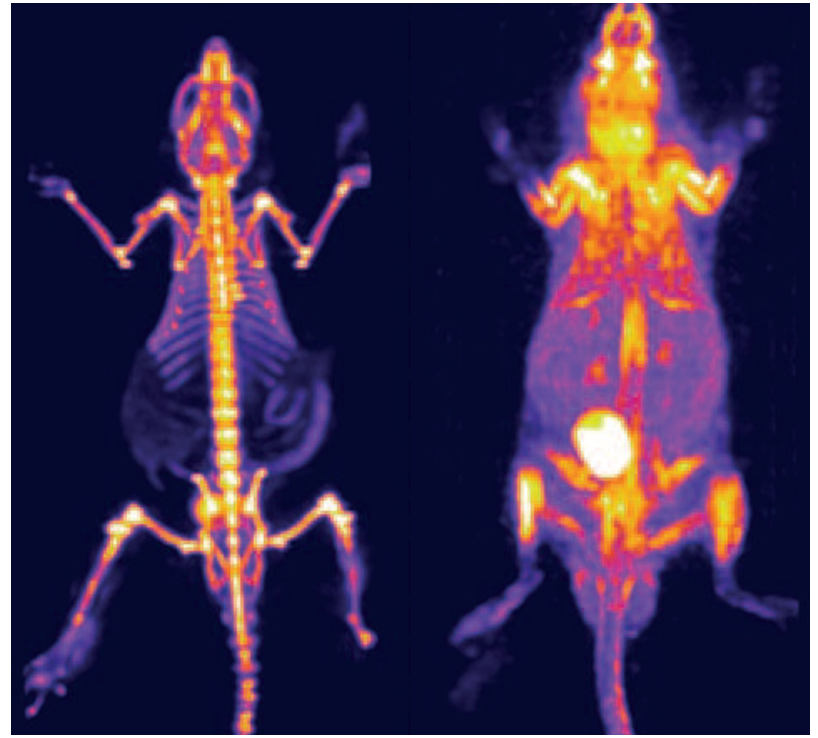

FIG. 6.-Imágenes MIP (Maximum Intensity Projection) $c{ }^{18} \mathrm{~F} y$ $F D G$ de ratones de 30 gramos, reconstruidas con un algoritmo iterativo 3D. La resolución de estos estudios, representativa de la máxima que se puede obtener hoy en día, está aún lejos de escalar a la de estudios en humanos. (Imágenes adquiridas con el sistema eXplore VISTA, GE Healthcare.)

sos es necesario recurrir a técnicas de microcirugía para la inserción de catéteres que permitan la extracción, aunque esto aumenta la morbilidad del animal en estudios longitudinales. Otra alternativa para la estimación de la función de entrada puede ser la realización de estudios dinámicos de gran velocidad, principalmente durante la parte inicial del experimento. En este caso, las curvas generadas a partir del análisis regional de la secuencia de imágenes junto con una adecuada calibración del sistema, pueden permitir una estimación precisa de dicha función de entrada. Esta técnica, relativamente sencilla de realizar en sistemas clínicos, conduce al límite de sus prestaciones a los sistemas de imagen animal, y por tanto dificulta su ejecución. De ahí la ventaja de contar con sistemas con múltiples protocolos de adquisición programables y la posibilidad de incorporar varias señales fisiológicas durante el proceso de obtención de imagen, con el fin de poder realizar estudios a posteriori con diversos criterios de sincronización.

\section{Limitaciones farmacológicas}

Las interacciones entre trazadores y los distintos tipos de anestesia, junto con los condicionantes impuestos por la dieta alimenticia o incluso por el pro- pio modelo de enfermedad del animal dificultan el diseño experimental. A esto se añade la necesidad de disponer de una elevada actividad específica en el trazador para mantener dentro de límites razonables el volumen de radiofármaco inyectado: es el denominado efecto de masa, que se explica más adelante ${ }^{45}$.

Los radiofármacos utilizados en PET pueden clasificarse en tres grandes grupos ${ }^{46}$ :

1. Aquéllos que marcan sistemas no saturables, (ejemplo: $\left[{ }^{18} \mathrm{~F}\right]$ en hueso).

2. Marcadores de sistemas medianamente saturables (ejemplo: $\left[{ }^{18} \mathrm{~F}\right]-F D G$ para la monitorización de cambios en la actividad de la GLUT o de la hexokinasa, o la $\left[{ }^{18} \mathrm{~F}\right]-\mathrm{FMT}$ utilizada en la visualización del sistema dopaminérgico).

3. Sistemas fácilmente saturables (ejemplo: $\left[{ }^{18} \mathrm{~F}\right]$-FCWAY, utilizado para la medida de densidad de receptores $5-\mathrm{HT}_{1 \mathrm{~A}}$ ).

El factor determinante de la calidad de imagen es el número de cuentas acumuladas por píxel (vóxel), que a su vez depende de la actividad acumulada en la zona de interés. Mientras que el uso de dosis altas para la visualización de sistemas no saturables no presenta mayores inconvenientes y se pueden obtener imágenes aceptables con prácticamente cualquier tomógrafo, la visualización de densidad de receptores supone una seria dificultad dada la limitación de la dosis, lo que deja como únicas alternativas el aumento de la actividad específica del trazador y el aumento de la sensibilidad del sistema de imagen.

Por lo tanto, los parámetros relevantes a considerar son: la cantidad mínima de actividad que se puede detectar, el número de receptores estimados en el tejido a visualizar, el tamaño del órgano, y la dosis de radiación a la que se expone la muestra. Se puede demostrar que un escalado de dosis lineal en función de la masa del sujeto del experimento, que sería correcto en términos farmacocinéticos para mantener la misma concentración en el humano y en el animal, no basta para conseguir la misma la calidad de imagen. Empleando simplemente el peso como factor de escala, la radioactividad inyectada a un modelo en rata se escalaría aproximadamente con un coeficiente $\sim 0,4 \%$ según el peso total $(0,25 \mathrm{~kg} / 70 \mathrm{~kg}), \mathrm{o} \sim 0,1 \%$ según la relación de peso entre ambos cerebros $(1.500 \mathrm{mg} / 1.500 \mathrm{~g})$.

Sin embargo, si se mantiene la proporción entre el peso del cerebro y el tamaño del vóxel, éste último 
debería tener en la imagen animal 1/1.000 del tamaño usado en humanos, lo que implica que necesitaríamos una actividad 1.000 veces mayor dentro de dicho vóxel para obtener la misma relación entre la señal y el fondo (es decir, número de cuentas que provienen de dicho vóxel de la estructura diana frente a la actividad inespecífica). Vemos como la aparente reducción de dosis derivada del escalado lineal de peso se compensa con la necesidad de mayor concentración de trazador por la disminución del tamaño del vóxel, lo que implica que la cantidad total de trazador (en cuentas) que habría que suministrar a la rata es la misma que la del humano. Dada la limitación en el volumen que es posible inyectar al animal, la solución al problema pasaría por el aumento de la actividad específica del trazador.

Para este cálculo teórico hemos supuesto que la resolución del escáner animal se escala en la misma proporción que la del humano. Sin embargo, la tecnología actual dista mucho de lograr esas resoluciones submilimétricas por culpa de las limitaciones físicas e instrumentales anteriormente expuestas ${ }^{21}$.

Un análisis matemático de este problema nos proporciona información más concreta; supongamos que la actividad contenida en un vóxel sea la misma en el estudio humano y en el animal, lo que se puede expresar como

$$
\begin{aligned}
& S_{\mathrm{r}} \cdot \mathrm{C}_{\mathrm{r}} \cdot \mathrm{V}_{\mathrm{r}} \cdot \rho_{\mathrm{r}} \cdot \mathrm{E}_{\mathrm{r}} \cdot \exp \left[-\mathrm{u} \cdot \mathrm{x}_{\mathrm{r}}\right]= \\
& =\mathrm{S}_{\mathrm{h}} \cdot \mathrm{C}_{\mathrm{h}} \cdot \mathrm{V}_{\mathrm{h}} \cdot \rho_{\mathrm{h}} \cdot \mathrm{E}_{\mathrm{h}} \cdot \exp \left[-\mathrm{u} \cdot \mathrm{x}_{\mathrm{h}}\right]
\end{aligned}
$$

donde el subíndice " $r$ " indica roedor y el subíndice "h" indica humano, y siendo S la actividad específica del trazador en $\mathrm{Ci} / \mathrm{g}, C$ la concentración de radiofármaco en $\mathrm{g} / \mathrm{g}, V$ el volumen del vóxel en cc, $\rho$ la densidad tisular en $\mathrm{g} / \mathrm{cc}, u$ el coeficiente de atenuación del tejido a $511 \mathrm{keV}$ en $\mathrm{cm}^{-1}, x_{h}$ y $x_{r}(\mathrm{~cm})$ el grosor típico de la muestra en la localización del vóxel bajo estudio, y $E$ la fracción de coincidencias detectadas por el sistema PET en relación con las desintegraciones realmente ocurridas (sensibilidad).

Esta ecuación permite el planteamiento de diferentes hipótesis de trabajo, y de su resolución se obtienen conclusiones en principio no intuitivas. Suponiendo que los tiempos de imagen son aproximadamente los mismos en todos los casos, que en ningún momento la dosis administrada satura el sistema bajo estudio, y que la densidad del tejido biológico es igual en el roedor y en el humano, despejando la ecuación para $C_{r}$ se obtiene:

$$
\mathrm{C}_{\mathrm{r}}=\mathrm{C}_{\mathrm{h}} \cdot \frac{\mathrm{S}_{\mathrm{h}}}{\mathrm{S}_{\mathrm{r}}} \cdot \frac{\mathrm{V}_{\mathrm{h}}}{\mathrm{V}_{\mathrm{r}}} \cdot \frac{\mathrm{E}_{\mathrm{h}}}{\mathrm{E}_{\mathrm{r}}} \cdot \exp \left[-\mathrm{u} \cdot\left(\mathrm{T}_{\mathrm{h}}-\mathrm{T}_{\mathrm{r}}\right)\right]
$$

Para una sección de cabeza humana de $18 \mathrm{~cm}$ frente a $3 \mathrm{~cm}$ en el caso de de la rata, con un coeficiente de atenuación lineal para el tejido biológico de $0.08 \mathrm{~cm}^{-1}$, se obtiene que el término exponencial es igual a 0,3. Escalando el tamaño de vóxel de forma proporcional a la relación de masas $(70 \mathrm{~kg}$ frente a $250 \mathrm{gm}$ ) se obtiene un factor 280. Si, por último, se considera que las eficiencias del detector para humanos y para animales son equivalentes, la expresión anterior se reduce a

$$
C_{r}=84 \cdot C_{h}
$$

La conclusión que se extrae es que, si se pretende usar el mismo trazador con idéntica actividad específica en el humano y en la rata, la concentración necesaria en el animal para mantener una relación señal a ruido similar en ambos casos resulta ser 84 veces mayor. Si esto no fuese posible y hubiese que mantener la misma concentración en los dos casos, entonces, asumiendo los mismos condicionantes que en el caso anterior, la ecuación se traduce en

$$
\frac{\mathrm{S}_{\mathrm{r}} \cdot \mathrm{E}_{\mathrm{r}}}{\mathrm{S}_{\mathrm{h}} \cdot \mathrm{E}_{\mathrm{h}}}=84
$$

expresión que indica la necesidad de mantener la relación entre el producto de la actividad específica y la sensibilidad del sistema, que ha de ser 84 veces más grande en el caso de la rata. Este factor es muy alto para la tecnología actual tanto de trazadores como del sistema de imagen, que en los mejores equipos disponibles en el mercado mejoran la sensibilidad con respecto a los sistemas de humanos en un factor 5 , dejando el resto para la mejora de la actividad específica del trazador. Como evidentemente este aumento puede no ser alcanzable según el trazador de que se trate, si se plantea la hipótesis de utilizar un sistema de imagen y un trazador que dejen esta relación igual a 1, la alternativa que queda es la de aumentar la cantidad total de radioactividad administrada al animal, que despejando da como resultado

$$
A_{r}=0,3 \cdot A_{h}
$$

Esto indica que la equivalencia en calidad de imagen se obtiene administrando al animal una dosis equivalente al $30 \%$ de la dosis humana, cantidad que 
Tabla 3

RESUMEN COMPARATIVO DE SISTEMAS COMERCIALES DE PET PARA PEQUEÑOS ANIMALES DE LABORATORIO. VER TEXTO PARA ACLARACIONES SOBRE LOS PARÁMETROS

\begin{tabular}{|c|c|c|c|c|c|}
\hline & \multirow{2}{*}{ Resolución FBP (mm) } & \multirow{2}{*}{ Sensibilidad (cps/ $\mu \mathrm{Ci})$} & \multirow{2}{*}{ Centelleador } & \multicolumn{2}{|c|}{ FOV } \\
\hline & & & & $\overline{A x i a l}(\mathrm{~mm})$ & Transaxial $(\mathrm{mm})$ \\
\hline GEMS eXplore Vista & $\begin{array}{l}1,6 \\
2,0(15 \mathrm{~mm} \text { off axis }) \\
2,5(30 \mathrm{~mm} \text { off axis })\end{array}$ & $1.554(4,2 \%)^{a}$ & LSO-GSO & 46 & 60 \\
\hline Concorde R4 & $\leq 1,8($ centro FOV $)$ & $1.480(4,0 \%)^{b}$ & LSO & 78 & 100 \\
\hline Concorde Focus 120 & $\leq 1,3($ centro FOV $)$ & $2.405(6,5 \%)^{b}$ & LSO & 76 & 100 \\
\hline HIDAC & 0,95 & $329(0,9 \%)$ & - & 210 & $100^{c}$ \\
\hline MOSAIC & $\begin{array}{l}2,3 \\
3,0 \text { (40 mm off axis) }\end{array}$ & $925(2,5 \%)$ & GSO & 118 & 180 \\
\hline COMECER (YA-(S)P PET) & $<1,8$ & $>629(1,7 \%)^{d}$ & YAP:Ce & 40 & 40 \\
\hline SUINSA rPET & 1,8 (centro FOV) & $>370(1,4 \%)^{b}$ & MLS & 48 & 48 \\
\hline
\end{tabular}

${ }^{a}$ Medido con la ventana de energía de $250-700 \mathrm{KeV}$.

${ }^{b}$ Medido con la ventana de energía de $250-700 \mathrm{KeV}$ y con $10 \pm 0,2$ ns de ventana de tiempo.

${ }^{c}$ Ajustable a $200 \mathrm{~mm}$.

${ }^{d}$ Sin ventana de energía.

en principio puede parecer muy exagerada si se compara con el resultado obtenido mediante el simple escalado proporcional a las masas.

La calidad de imagen es función de la cantidad de cuentas acumuladas y no de la tasa de conteo instantánea (la tasa de coincidencias válidas aumenta linealmente con la dosis, mientras que la tasa de coincidencias aleatorias aumenta con el cuadrado de ésta ${ }^{1}$ ), y que por lo tanto se pueden alcanzar diferentes compromisos entre dosis y duración del estudio, de forma que al final del mismo se acumulen la misma cantidad de cuentas. Esto es especialmente interesante cuando se esté trabajando con sistemas saturables.

También ocurre que, en pequeños animales, zonas de interés como puede ser el corazón, se encuentran cerca de otros órganos de nulo interés pero de muy alta acumulación de actividad, como por ejemplo puede ser la vejiga, y en consecuencia aparece un aumento de la fracción de eventos dispersos que se acumulan en el estudio, lo cual es importante en sistemas de imagen con campos de visión axial grandes que incluyen el animal entero bajo el área de imagen ${ }^{47}$.

\section{EQUIPOS COMERCIALES}

En esta sección se hace un breve repaso de algunos sistemas disponibles comercialmente; los datos aquí reflejados se han obtenido de fuentes heterogéneas: catálogos comerciales, publicaciones y páginas de Internet de los fabricantes. Es importante advertir sobre la dificultad de establecer comparaciones directas ya que los resultados no suelen corresponder a unas condiciones de medida similares (ver detalles metodológicos en las referencias) y por lo tanto no reflejan las mismas características. Un análisis fiable de estos datos queda pendiente hasta la publicación del protocolo NEMA.

Existen dos grandes clases de sistemas: sistemas que consisten en detectores parciales, y que por lo tanto han de girar alrededor de la muestra ${ }^{29,48,49}$, y los de anillo completo. La razón que justifica la existencia de estas dos aproximaciones tecnológicas es de índole económica, dado que el componente más caro de todo el sistema es el detector, y completar un anillo con el fin de eliminar la necesidad del movimiento de rotación es sin duda una solución costosa (tabla 3 ).

\section{Concorde Microsystems}

Concorde Microsystems es una compañía ligada a CTI, fabricante de sistemas para humanos, para los cuales diseña y fabrica componentes electrónicos (los circuitos ASICS que incorporan esos sistemas). La familia microPET (marca registrada por esta compañía) surgió como una transferencia de tecnología del grupo del Instituto Crump de UCLA, en donde se de- 
sarrollaron los primeros prototipos ${ }^{50}$. Es la marca que lleva más tiempo en el mercado, y en los últimos meses han añadido a su catálogo de productos una nueva línea denominada FOCUS que introduce mejoras sobre la resolución del sistema original ${ }^{51}$. Se trata de equipos de anillo completo basados en matrices de cristales de centelleo LSO pixelados y acoplados mediante guías de luz de fibra óptica a tubos fotomultiplicadores sensibles a posición. Detalles sobre estos sistemas pueden encontrarse en http://concorde.ctimi.com/.

\section{HiDAC}

Los sistemas de Oxford Positron Systems (http:// www.oxpos.co.uk/) denominados HiDAC utilizan como detector cámaras de avalancha de alta densidad, tecnología que no usa ni centelleadores ni fotodetectores. Estos detectores fueron diseñados originalmente para experimentos en física de altas energías en el CERN, tienen un tamaño relativamente grande comparado con el resto de sistemas en el mercado, $\left(20 \times 20 \mathrm{~cm}^{2}\right)$ y se agrupan en bancos proporcionando un gran campo de visión de $10 \mathrm{~cm}$ de longitud y $10 \mathrm{~cm}$ de diámetro ${ }^{52,53}$. Debido a su especial diseño, sólo pueden utilizar técnicas estadístico-iterativas para la reconstrucción de imagen.

\section{eXplore VISTA}

Este equipo, fabricado originalmente por la compañía española SUINSA con el nombre de ARGUS, surgió de una transferencia tecnológica de la empresa norteamericana TRIDENT y del laboratorio de imagen de la Unidad de Medicina Experimental del Hospital Gregorio Marañón de Madrid.

Actualmente está comercializado por la empresa General Electric Heathcare bajo el nombre de eXplore VISTA (http://www.gehealthcare.com/usen/ fun_img/pcimaging/products/vista.html). Utiliza un anillo completo de fotomultiplicadores sensibles a posición, con un campo de visión axial algo menor que el de otros sistemas similares ${ }^{25,26,54}$. Ofrece la particularidad de ser el único equipo comercializado que utiliza doble capa de cristales (phoswich) en los detectores para reducir el efecto de la DOI, lo que proporciona una baja degradación de resolución al alejarse del centro. Se ofrece la posibilidad de PET-CT combinado, utilizando el CT eXplore Locus de la misma compañía.

\section{MOSAIC}

El sistema MOSAIC de Philips es heredero de la tecnología desarrollada en la Universidad de Pensilvania, reciclando la última generación de detectores diseñados por este grupo para los sistemas de humanos (Pixelar), y adaptándola a la problemática del la imagen en pequeños animales. El resultado es un sistema con un gran campo de visión axial que usa cristales de GSO y fotomultiplicadores no sensibles a posición, lo que limita su resolución a más de $2 \mathrm{~mm}$. Por ser un sistema de reciente aparición apenas se dispone de resultados en la literatura científica que permitan evaluar sus prestaciones ${ }^{55}$. Será interesante ver las imágenes de rata y ratón que produzca este sistema dada la muy particular tecnología de este diseño (uso de GSO como centelleador único y campo axial grande con relación al tamaño de la muestra). El fabricante también anuncia la próxima disponibilidad de CT-PET combinado.

\section{YAP-(S)PET}

Las empresas italianas ISE y COMECER comercializan un sistema surgido de la Universidad de Ferrara, denominado YAP-(S)PET, que tiene la especial característica de poder funcionar como PET o como SPECT. Se trata de un sistema de detectores giratorios en cristales pixelados de YAP:Ce acoplados a fotomultiplicadores sensibles a posición ${ }^{48}$. A pesar de tratarse de un centelleador de baja densidad, puede ser usado para la detección de fotones gamma de $511 \mathrm{keV}$, utilizando la interacción Compton en vez de la fotoeléctrica, lo que limita su resolución en energía; si se inserta un colimador también puede usarse para trazadores de fotón único de menor energía.

\section{rPET}

La empresa española SUINSA antes mencionada, también en colaboración con la Unidad de Medicina y Cirugía Experimental del Hospital Gregorio Marañón, ha desarrollado recientemente un sistema basado en detectores planos opuestos rotatorios ${ }^{18,56}$. Como centelleador se usa cristal de oxiortosilicato de lutecio (MLS), con características de emisión de luz y densidad efectiva similares al LSO. El sistema es modular, lo que permite ajustar su coste eligiendo diferentes niveles de sensibilidad e integrar otras modalidades de imagen (SPECT y CT) en una geometría 
coplanar, que no requiere mover la muestra para obtener datos combinados PET-CT o SPECT-CT.

Existen otros sistemas de muy reciente aparición basados en fotodetectores semiconductores (Advanced Molecular Imaging, Inc., Canada), o sistemas multimodales SPECT/CT/PET (GammaMedica, Inc., USA) para los cuales todavía no se dispone de datos.

\section{BIBLIOGRAFÍA}

1. Chatziioannou AF. Molecular Imaging of Small Animals with Dedidacted PET Tomographs. Eur J Nucl Med. 2002;29: 98-114.

2. Weissleder R. Scaling down imaging: molecular mapping of cancer in mice. Nat Rev Cancer. 2002;2:11-8.

3. Lewis JS, Achilefu S. Small animal imaging: Current technology and perpectives for oncological imaging. Eur J Cancer. 2002; 38:2173-88.

4. Phelps ME. The merging of biology and imaging into molecular imaging. J Nucl Med. 2000;41:661-81.

5. Massoud TF, Gambhir SS. Molecular imaging in living subjects: seeing fundamental biological processes in a new light. Gene Dev. 2003; 17:545-80.

6. Pomper MG. Molecular imaging and overview. Acad Radiol. 2001;8:1141-53.

7. Vaquero JJ, Seidel J, Lee I, Green MV. High resolution imaging in small animals with positron emission tomography and other modalities. Lab Anim. 2000:147-50.

8. Cherry SR. In vivo molecular and genomic imaging: new challenges for imaging physics. Phys Med Biol. 2004;49:R13-R48.

9. Desco M, editor. Monográfico: Tomografía por emisión de positrones (PET); 2002.

10. Hoffman EJ, Phelps ME. Positron emission tomography: Principles and quantitation. En: Phelps ME, Mazziotta JC, Schelbert HR, editors. Positron emission tomography and autoradiography. New York: Raven Press; 1986. p. 237-86.

11. Budinger TF, Derenzo SE, Huesman RH. Instrumentation for positron emission tomography. Ann Neurol. 1984;15:S35-S43.

12. Jone T. Positron emission tomography. Clinical Physics and Physiological Measurement (York). 1990;11A:27-36.

13. Ollinger JM, Fessler JA. Positron-emission tomography. IEEE Signal Proc Mag. 1997;14:43-55.

14. Zanzonico P. Positron emission tomography: A review of basic principles, scanner design and performance, and current systems. Semin Nucl Med. 2004;34:87-111.

15. NEMA_Task_force_draft. Performance Measurements of Positron Emission Tomographs for Laboratory Animals: National Electrical Manufacturers Association; 2001.

16. Daube-Witherspoon ME. Acceptance testing, maintenance and quality control of PET instrumentation, in PET/SPECT: instrumentation, radiopharmaceuticals, neurology and physiological measurement. Washington: American College of Nuclear Physicians; 1988. p. 60-71.

17. Brix G, Zaers J, Adam LE, Bellemann ME, Ostertag H, Trojan $\mathrm{H}$, et al. Performance evaluation of a whole-body PET scanner using the NEMA protocol. J Nucl Med. 1997;38:1614-23.

18. Vaquero JJ, Desco M, Pascau J, Santos A, Lee IJ, Seidel J, et al. PET, CT and MR image registration of the rat brain and skull. IEEE T Nucl Sci 2001;48:1440-5.
19. Kinahan PE, Townsend DW, Beyer T, Sashin D. Attenuation correction for a combined 3D PET/CT scanner. Med Phys. 1998; 25:2046-53.

20. Lecomte R. Technology challenges in small animal PET imaging. Nucl Instrum Methods Phys Res. 2004;527:157-65.

21. Levin CS, Hoffman EJ. Calculation of positron range and its effect on the fundamental limit of positron emission tomography system spatial resolution. Phys Med Biol. 1999;44:781-99.

22. Phelps ME, Hoffman EJ, Huang SC, Ter-Pogossian MM. Effect of positron range on spatial resolution. J Nucl Med. 1975;16: 649-52.

23. Melcher CL. Scintillation crystals for PET. J Nucl Med. 2000; 41:1051-5.

24. Seidel J, Gandler WR, Green MV. Characteristics of a pair of small Field-of-View LSO scintillation cameras. IEEE T Nucl Sci. 1996;43:1968-73.

25. Seidel J, Vaquero JJ, Green MV. Resolution uniformity and sensitivity of the NIH ATLAS small animal PET scanner: comparison to simulated LSO scanners without Depth-of-Interaction capability. IEEE T Nucl Sci. 2003;50:1347-50.

26. Seidel J, Vaquero JJ, Pascau J, Desco M, Johnson CA, Green MV. Features of the NIH atlas small animal pet scanner and its use with a coaxial small animal volume CT scanner. Proc IEEE International Symposium on Biomedical Imaging. 2002: 545-8.

27. Ziegler SI, Pichler BJ, Boening G, Rafecas M, Pimpl W, Lorenz $\mathrm{E}$, et al. A prototype high-resolution animal positron tomograph with avalanche photodiode arrays and LSO crystals. Eur J Nucl Med. 2001;28:136-43

28. Vaquero JJ, Seidel J, Siegel S, Gandler WR, Green MV. Performance Characteristics of a Compact Position-Sensitive LSO Detector Module. IEEE T Med Imaging. 1998;17:967-78.

29. Siegel S, Vaquero JJ, Aloj L, Seidel J, Jagoda E, Gandler WR, et al. Initial results from a PET/Planar small animal imaging system. IEEE T Nucl Sci. 1999;46:571-5.

30. Miyaoaka RS, Kohlmyer SG, Lewellen TK. A second generation micro crystal element (MICE2) detector. J Nucl Med. 2001; 42:102P.

31. Johnson CA, Seidel J, Carson RE, Gandler WR. Evaluation of 3D reconstruction algorithms for a small animal PET camera. IEEE T Nucl Sci. 1997;44:1303-8.

32. Muehllehner G, Karp JS. A positron camera using position-sensitive detectors: PENN-PET. J Nucl Med. 1986;27:90-8.

33. Ter-Pogossian MM, Raichle ME, Sobel BE. Positron-emission tomography. Sci Amer. 1980;243:170-81.

34. Inoue K, Nagai Y, Saito H, Nagashima Y, Hyodo T, Muramatsu $\mathrm{S}$, et al. A position-sensitive scintillation detector for two-dimensional angular correlation of annihilation radiation using metal-package position-sensitive photomultiplier tubes. Nucl Instrum Methods Phys Res A. 1999;423:364-8.

35. Correia JA, Burnham CA, Kaufman D, Fischman AJ. Development of a small animal PET imaging device with resolution approaching $1 \mathrm{~mm}$. IEEE T Nucl Sci. 1999;46:631-5.

36. Chatziioannou AF, Cherry SR, Shao Y, Silverman RW, Meadors K, Farquhar TH, et al. Performance evaluation of microPET: A high-resolution Lutetium Oxyorthosilicate PET scanner for animal imaging. J Nucl Med. 1999;40:1164-75.

37. Cherry SR. Recent advances in instrumentation for positron emission tomography. Nucl Instrum Methods Phys Res. 1994; 348:577-82.

38. Weber DA, Ivanovic M. Ultra-high-resolution imaging of small animals: Implications for preclinical and research studies. J Nucl Cardiol. 1999;6:332-44.

39. Weisenberger AG, Bradley EL, Majewski S, Saha MS. Development of a novel radiation imaging detector system for in vivo 
gene imaging in small animal studies. IEEE T Nucl Sci. 1998; 45:1743-9.

40. Lecomte R, Cadorette JE, Rodrigue S, Lapointe D, Rouleau D, Bentourkia $M$, et al. Initial results from the Sherbrooke avalanche photodiode positron tomograph. IEEE T Nucl Sci. 1996;43:1952-7.

41. Defrise M, Kinahan PE, Townsend DW. Exact and approximate rebinning algorithms for 3D-PET data. IEEE T Med Imaging. 1997; 16:145-58.

42. Defrise M, Townsend DW, Geissbuhler A. Implementation of three-dimensional image reconstruction for multi-ring positron tomographs. Phys Med Biol. 1990;35:1361-72.

43. Brasse D, Kinahan PE, Clackdoyle R, Defrise M, Comtat C, Townsend DW. Fast fully 3-D image reconstruction in PET using planograms. IEEE T Med Imaging. 2004;23:413-25.

44. Lapoite D, cardorete J, Rogdriguez S, Rouleau D, Lecomte R. A microvolumetric blood counter/sampler for metabolic PET studies in small animals. IEEE T Nucl Sci. 1998;45:2195-9.

45. Jagoda EM, Vaquero JJ, Seidel J, Green MV, Eckelman WC. Experiment assessment of mass effects in the rat: implications for small animal PET imaging. Nucl Med Biol. 2004;31:771-9.

46. Gambhir SS, Czernin J, Schwimmer J, et al. A tabulated summary of the FDG PET literature. J Nucl Med. 2001;42:1S-93S.

47. Strother SC, Casey ME, Hoffman EJ. Measuring PET scanner sensitivity: relating countrates to image signal-to-noise rataios using Noise Equivalent Counts. IEEE T Nucl Sci. 1990;37:783-8.

48. Humm JL, Rosenfeld A, Del Guerra A. From PET detectors to PET scanners. Eur J Nucl Med Mol Imaging. 2003;30:1574-97.
49. Townsend D, Byars L, Defrise M, Geissbuhler A, Nutt R. Rotating positron tomographs revisited. Phys Med Biol. 1994; 39:401-10.

50. Cherry SR, Shao Y, Silverman RW, Meadors K. MicroPET: A high resolution PET scanner for imaging small animals. IEEE T Nucl Sci. 1997;44:1161-6.

51. Tai YC, Ruangma A, Rowland D, Siegel S, Newport DF, Chow PL, et al. Performance evaluation of the microPET Focus: A third-generation microPET scanner dedicated to animal imaging. J Nucl Med. 2004;46:455-68.

52. Jeavons AP, Chandler RA, Dettmar CAR. A 3D HIDAC-PET Camera with Sub-millimetre Resolution for Imaging Small Animals. IEEE T Nucl Sci. 1999;46:468-73.

53. Missimer J, Madi Z, Honer M, Keller C, Schubiger A, Ametamey SM. Performance evaluation of the 16-module quad-HIDAC small animal PET camera. Phys Med Biol. 2004:49:2069-81.

54. López J, España S, Udías JM, Vaquero JJ, Seidel J, Desco M. Full 3D-OSEM reconstruction with compressed response of the system. Book of Abstracts of 2004 IEEE Nuclear Science Symposium and Medical Imaging Conference. 2004. p. 163.

55. Huisman M, Weber A, Zigler S, Hamisch Y, Paulus T, Schwaiger M. Performance evaluation and system characteristics of the Philips 'Mosaic' animal positron emission tomography system. Mol Imaging Biol. 2005;7:135.

56. Vaquero JJ. Imagen Molecular. En: García-Barreno P, Espinós-Pérez D, Cascales-Angosto M, editors. Cáncer. Madrid: Instituto de España; 2003. p. 283-310. 